\title{
Optical Properties of Aligned Nematic Liquid Crystals in Electric Field
}

\author{
Suleyman Yilmaz ${ }^{1}$, Halide Melik ${ }^{1}$, Firat Angay ${ }^{1}$, Mehriban Emek ${ }^{2}$, Ahmet Yildirim ${ }^{3}$ \\ ${ }^{1}$ Department of Physics, Harran University, Osmanbey Campus, Sanliurfa, Turkey \\ ${ }^{2}$ Department of Physics, Adiyaman University, Adiyaman, Turkey \\ ${ }^{3}$ Department of Physics, Siirt University, Siirt, Turkey \\ E-mail: syilmaz@harran.edu.tr \\ Received January 13, 2011; revised February 23, 2011; accepted February 25, 2011
}

\begin{abstract}
In this study, the optical transmittance of aligned nematic liquid crystals (ANLCs) was investigated in terms of temperature variations through electrooptic effects under DC electric field. The optical transmittances of the planar and homeotropically aligned liquid crystal cells, which were prepared by conventional rubbing and photolithographic technique on the polyimide thin films for molecular alignment, were measured in their phase transition region. The results of measurement for both orientations, the distribution curve of the optical transmittance exhibits displacement toward to low level at the beginning and then to high level by the temperature variations, while the electric field increases. It was also observed that the domain structure of the materials were affected considerably by the applied electric field and phase transition region of the aligned structures had broader range than by the pure crystalline structure and its phase transition temperature was changed by the molecular anisotropy. Finally, in photolithographic method strong bonds between the molecules and the orienting surface were observed in high contrast to rubbing method.
\end{abstract}

Keywords: Liquid Crystals, Thin Films, Photolithography, Optical Properties

\section{Introduction}

The nature of the liquid crystals (LC) is very complex and their aligned surface is included the important factors such as van der Waals, dipolar and steric interactions, hydrogen and chemical bonding and surface topography. Their electrooptical properties also play important role on optical processing systems and photonic devices such as mobile phones, smart cards, integrated displays [1,2]. At the same time, NLCs are preferred in many different applications due to their anisotropic structure, which relates to molecular orientation and temperature variation [3-7].

The treatise on the surface of glass substrate constrains the LC and orients the surface director. The characteristic small area and multi domain properties concerned with molecular interactions and the other factors as well as the molecular anisotropy, the electrooptical effects, the anchoring energy, response time, contrast ratio etc.

There are several techniques for surface alignment for LCs that depending on contact and non-contact procedure. The rubbed polymer technique is widely used in
LCDs and the perfect electrooptic performance is provided by this method. However, the fiber residues, the corresponding impurities, the static charges and the mechanical damage can also trouble for devices and result in deterioration of the quality of LC switching, particularly when active matrix elements are used for the purpose [8]. Other alignment methods, such as photo- alignment, micron rubbing by microsphere, nanoim- printing lithography, microcontact printing and ion beam bombardment have also intensively studied.

In this study, the photolithography method was used to providing the micropatterns on the polyimide surface that includes the spin-coating, the UV exposure and the wet chemical etching, respectively. By taking into account the mentioned properties of NLCs, the optical and the electrooptical properties of randomly [9] and aligned NLCs are examined in the temperature range including phase transitions.

\section{Photolithographic Application}

In these study, commercial NLCs 5CB was provided 
from Sigma Aldrich, polyimide for planar alignment, adhesive material and thinner were provided from HD Microsystems, polyimide for homeotropic alignment and solvent were provided from Brewer Science, $\mathrm{NaOH}$ developer and positive photo-resist were provided from Rohm and Haas Electronic Materials.

The various materials used to aligned surface, but the polyimide is a suitable material for coating on the surface due to high cure temperatures $\left(\sim 350^{\circ} \mathrm{C}\right)$. Because of their excellent properties such as high refractive index, adhesion the substrates, high resistivity, excellent transparency in the visible spectrum, high chemical and thermal stability, they are usually preferred as the aligned surfaces.

On the experimental stage, firstly the adhesion promoter is performed on the indium tin oxide (ITO) glass substrates with resistance of $30-60 \Omega$ by using spin coater, after the polyimide coating, the photoresist material is coated on the surface of the polyimide film and finally the coated multilayer is exposed under periodic lined photomask by the UV light. Aligned multilayer surface is subjected to development procedure to removing contaminated materials from the surface and it is so called the wet chemical etching process. The alignment of surface was completed by curing with suitable heating and cooling procedure. The liquid crystal cells are formed with two anti-parallel aligned substrates and inserted into microfibers as spacer.

Morphologic texture of the NLC samples is obtained by using Leica polarization microscope with a CCD camera. The interferometric measurement system is designed by HeNe laser with $632.8 \mathrm{~nm}$, optical chopper, large area photoreceiver, lock in amplifier, other optical apparatus such as polarizer and analyzer. Experimental system is comprised of three units such as the heating, the electric field and the optical measurement unit (Fig- ure 1). The regular increments of the temperature were provided by the temperature control unit. In progressing system, the electric field was provided by the DC power supply to affect the molecular orientation. For determining the molecular orientation and anisotropy, the angle of polarizer was fixed in parallel position. The data obtained by the experimental system were transferred to a computer by the serial port and compiled by the Labview8 program.

\section{Optical Properties}

The NLCs are insulating organic liquids and formation of longitudinally oriented molecules. These structures have molecular orientation at certain temperature intervals as crystals have, but the gravity centers of the molecules are in chaotic order as in liquids. While there are no external alignment effects, there are randomly oriented domains in the nematic phase. However, if the domain walls of the liquid crystal are processed with known methods, the nematic liquid crystals will have the homogeneous single alignment $[9,10]$. The electrooptical effects in the LCs may be caused by two different physical processes: 1) the external electric field changes the orientation of molecules causing the absorption and scattering spectrum intensity, 2) the electric field changes the distances between energy levels of the molecule, shifts the absorption bands and varies the transition intensities. When the electric field is applied to such a system, the interaction between liquid crystal and electric field can be explained by means of at least two mechanisms [11]. The first one is the dependency of dielectric constant of LCs to molecular anisotropy $\left(\varepsilon_{\perp} \neq \varepsilon_{/ /}\right)$, this also implies that material is birefringence $\left(n_{\perp} \neq n_{/ /}\right.$or $\left.n_{o} \neq n_{e}\right)$. The contribution of this anisotropy mechanism to the system energy is as follows:

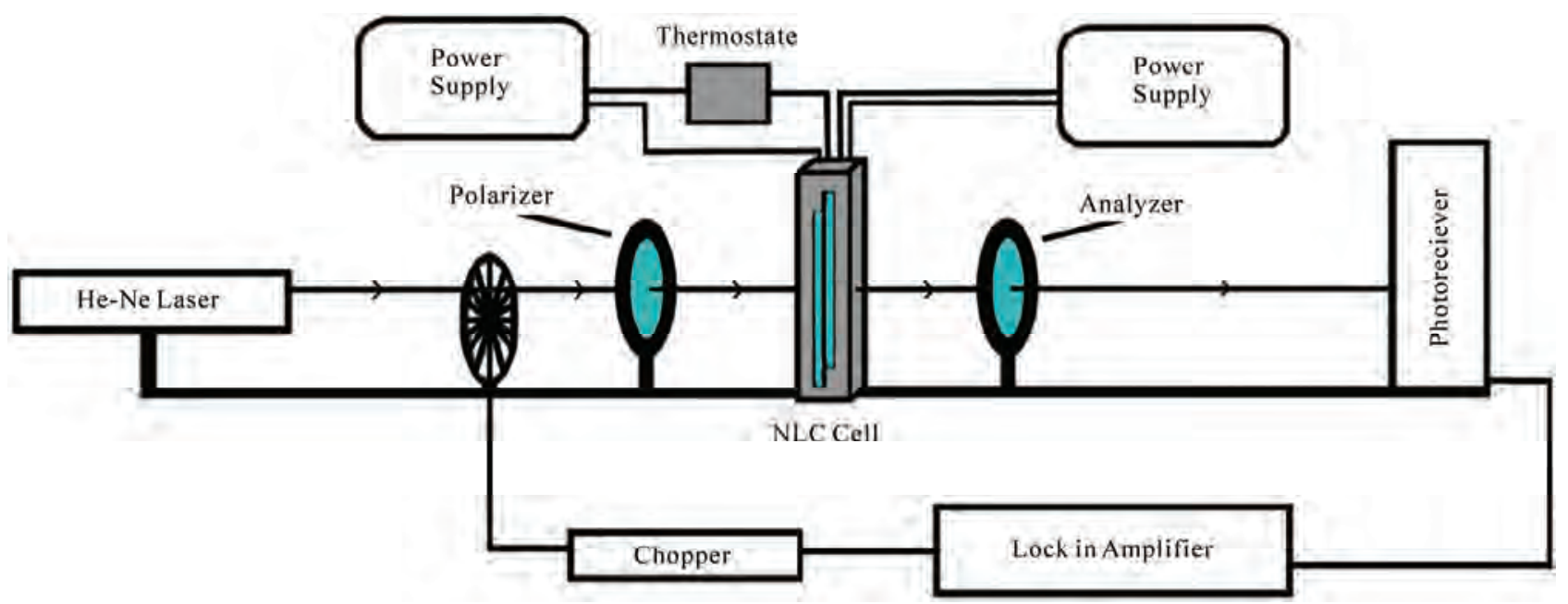

Figure 1. Schematic projection of the heating, the electrical field (DC) and the optical measurement units. 


$$
W_{e}=-\frac{1}{8 \pi} \varepsilon_{a}(\boldsymbol{n E})^{2}
$$

where, $\boldsymbol{n}$ is the director, $\boldsymbol{E}$ is the applied electric field and $\varepsilon_{a}=\varepsilon_{/ /}-\varepsilon_{\perp}$ dielectric anisotropy, $\varepsilon_{/ /}$and $\varepsilon_{\perp}$ denote parallel to and perpendicular constants, respectively. The second mechanism is similar to piezo-electric mechanism in solid materials, which small deformations cause formation of polarization. For a weak electric field, the mechanical tension forces are dominant and the orientation of molecular director remains the same. However, the orientation of director starts changing as the applied electric field rises over the critical value $\left(\boldsymbol{E}_{C}\right)$, which is known as Frederick's transition. To explain this process better, a function known as the coherence length for electric field is used;

$$
\zeta(\boldsymbol{E})=\left(\frac{4 \pi}{\varepsilon_{a}} K\right)^{1 / 2} \frac{1}{\boldsymbol{E}}
$$

where, $\zeta(E)$ denotes the coherence length that expresses the distance at which the director vector starts getting affected by the electric field. Here, $K$ is a constant denoting the mechanical tension. Thus, the critical electric field ( $\boldsymbol{E}_{C}$ ) is as follows:

$$
\boldsymbol{E}_{C} \cong \frac{1}{d}\left(\frac{4 \pi}{\varepsilon_{a}} K\right)^{1 / 2}
$$

As seen from (Equation (3)), the critical electric field depends on anisotropy ( $\varepsilon_{a}$ ) of the material and the thickness of the sample $(d)$. The transmittance of the liquid crystal cell is calculated as function of the applied electric field in two steps [12]: firstly, the reorientation of the molecular director must be determined in regards to applied electric field variations and secondly, the orientation of molecules affecting the transmittance of the light through the material must be calculated. These calculations are done by solving numerically the continuity equations for LCs [13]. Thus, anisotropy of molecular structure and the interaction of the incident light with the material determine the polarization of the transmitted light. If a polarization rotation is observed in material, one can tell about dichroism of the optically active material. The dichroism occurs in the LCs due to either the optical anisotropy of the molecular structure or the presence of the impurities. The dichroism also refers to any optical device which can split of light into beams with differing wavelengths.

When the light passes through the sample, the polarization state of the transmitted light is changed by molecular anisotropy [14]. In order to calculate the intensity of the light passing through a sample, Jones' vector representation is used [15]. The field components of the incident light perpendicular to each other are the follow- ing:

$$
\begin{aligned}
& \boldsymbol{E}_{x}=A_{x} \cos \left(\omega t-k z+\delta_{x}\right) \hat{\boldsymbol{i}} \\
& \boldsymbol{E}_{y}=A_{y} \cos \left(\omega t-k z+\delta_{y}\right) \hat{\boldsymbol{j}}
\end{aligned}
$$

The representation of the incident light components with the Jones' vector is the following:

$$
\boldsymbol{E}=\left(\begin{array}{l}
E_{x} \\
E_{y}
\end{array}\right)
$$

By the interaction of the transmitted light with the material, the components are the following:

$$
\begin{aligned}
& \boldsymbol{E}_{x}^{\prime}=A_{x}^{\prime} \cos \left(\omega t-k z+\delta_{x}^{\prime}\right) \hat{\boldsymbol{i}} \\
& \boldsymbol{E}_{y}^{\prime}=A_{y}^{\prime} \cos \left(\omega t-k z+\delta_{y}^{\prime}\right) \hat{\boldsymbol{j}}
\end{aligned}
$$

The representation of the transmitted light components with the Jones' vector is the following:

$$
\boldsymbol{E}^{\prime}=\left(\begin{array}{c}
E_{x}^{\prime} \\
E_{y}^{\prime}
\end{array}\right)
$$

Therefore, the optical transmittance of the LC cell system is calculated by the following equation:

$$
T=\frac{\left|E_{x}^{\prime}\right|^{2}+\left|E_{y}^{\prime}\right|^{2}}{\left|E_{x}\right|^{2}+\left|E_{y}\right|^{2}}
$$

\section{Results and Discussions}

The most common applications of NLC are seen in the electrooptic area depending on temperature. The optical transmittance of NLC 5CB is analyzed under DC electric field in terms of temperature variations. The relative transmittance for both the planar and the homeotropic orientation by the voltage values of $0-15 \mathrm{~V}$ is measured in the temperature range from $298 \mathrm{~K}$ to $328 \mathrm{~K}$ in Figures 2-4. The temperature increment of $3 \mathrm{~K}$ per minute is employed during the all measurement.

The devised LC systems in this study contain not only the conducting cell of the substrate glasses but also the optical attachments such as polarizer and analyzer. The variation of the anisotropy in the material due to the interaction between the material and light under the electric field is monitored and new optical modes are derived from these observations. An increase in the transparency of the sample depending on the temperature is clearly seen from the modulation spectrum graphics in Figures 5-7. The modulation spectrum data gives us detailed information about the optical properties of the material, such as the phase transition, the transparency and the molecular anisotropy. 


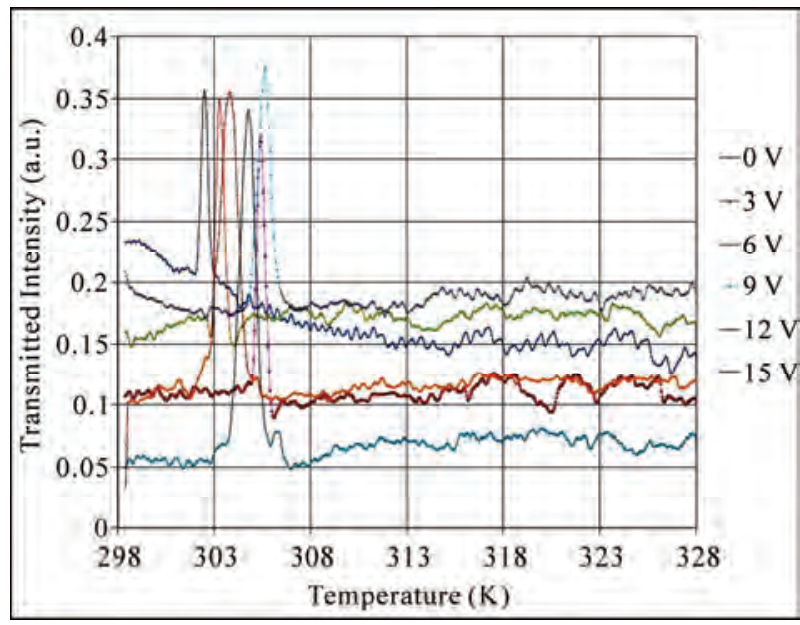

Figure 2. The transmitted intensity versus the temperature variations for the planar orientation by mechanical rubbing.

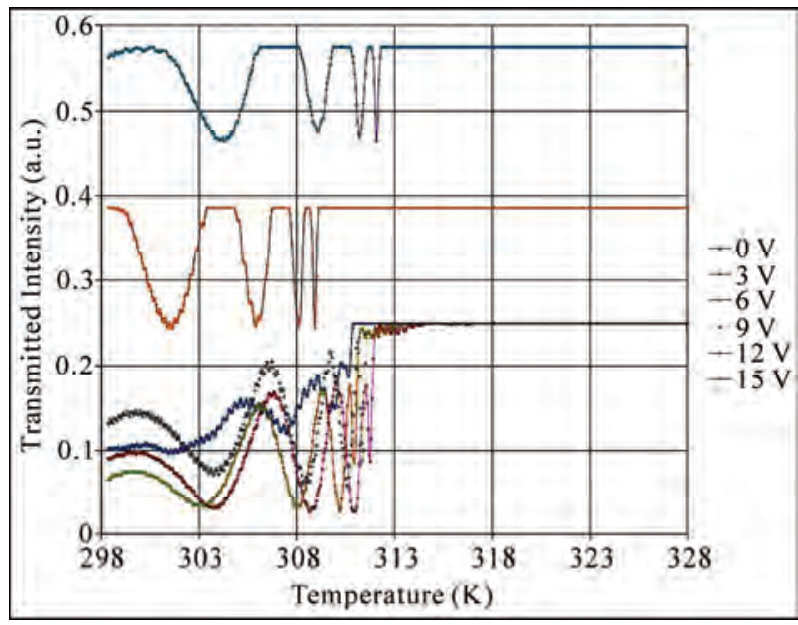

Figure 3. The transmitted intensity versus the temperature variations for the planar orientation by photolithography.

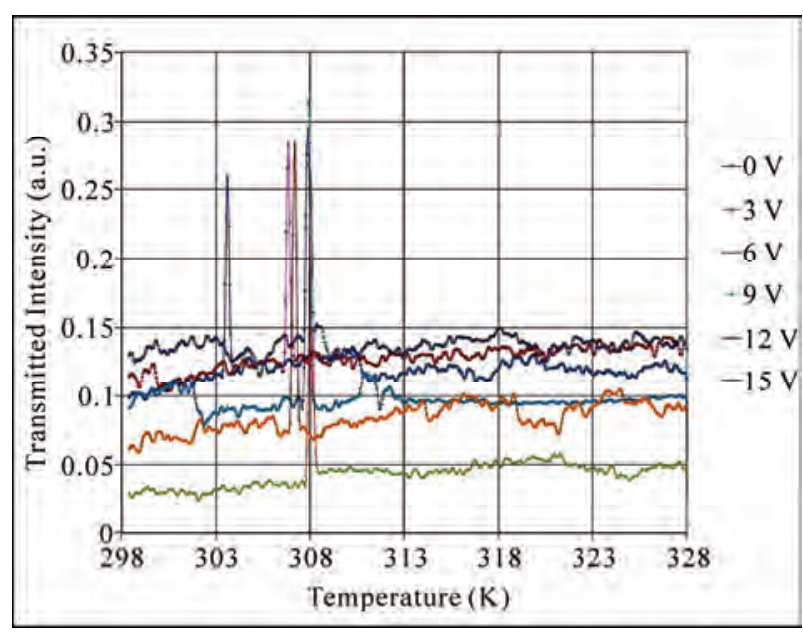

Figure 4. The transmitted intensity versus the temperature variations for the homeotropic orientation.
The analyzer angle is fixed $0^{\circ}$ (parallel to polarizer) and the LC cell is heated $3 \mathrm{~K}$ per minute in sample holder. At the beginning, the transmitted intensity exhibits a decreasing to low level and by the critical limit it reaches to high level on all of the molecular alignments in Figures 2-5. In the measurements for planar orientation by mechanical rubbing, the curves of the transmitted intensity exhibits firstly displacement toward to low level and it inclines to high level by the critical limit, $6 \mathrm{~V}$. At the photolithography application for planar alignment, the curves of the transmitted intensity exhibits firstly drastically displacement toward to low level and it inclines to high level by critical limit, $2.5 \mathrm{~V}$.

Finally, on the homeotropic alignment, the curves of the transmitted intensity also exhibits displacement toward to low level and it inclines to high level by the critical limit, $6 \mathrm{~V}$ like as planar alignment by mechanical rubbing.

The points at which the transmitted intensity reaches the maximum or the minimum value are seen at the modulation spectrum graphics in Figures 5-7. At the spectrum modulation graphics for all of the alignment application, the peak value of the transmitted intensity displaces to high temperature point step by step by the electric field variations. However, in Figure 6, the oscillations of the spectrum modulation for the photolithography application exhibit difference from the other previous alignments and by the critical point, they continue to their progress as smoothly.

\section{Conclusions}

In this presented study, the rubbing and photolithographic technique have been treated for molecular alignment and compared the results of photolithographic application with the conventional rubbing. When the electric field is applied, the electrooptical effects come into existence with variation on the molecular orientations; the electric field changes the anisotropy and leads to important effects on the processing of the optical materials.

It was observed that photolithography technique provides a controllable pretilt angle, strong anchoring strength (polar and azimuthal orientations), high contrast ratio of the electric field states (on and off) and response time by the low voltage driving as well as having high thermal and ultraviolet stability. It is interesting to note that there are meaningful and comparable results in experimenting under the DC electric fields.

Finally, the measurements that were carried out depending on the optical transmittance show that the phase transition interval of the aligned molecular structures was observed in larger temperature ranges. 

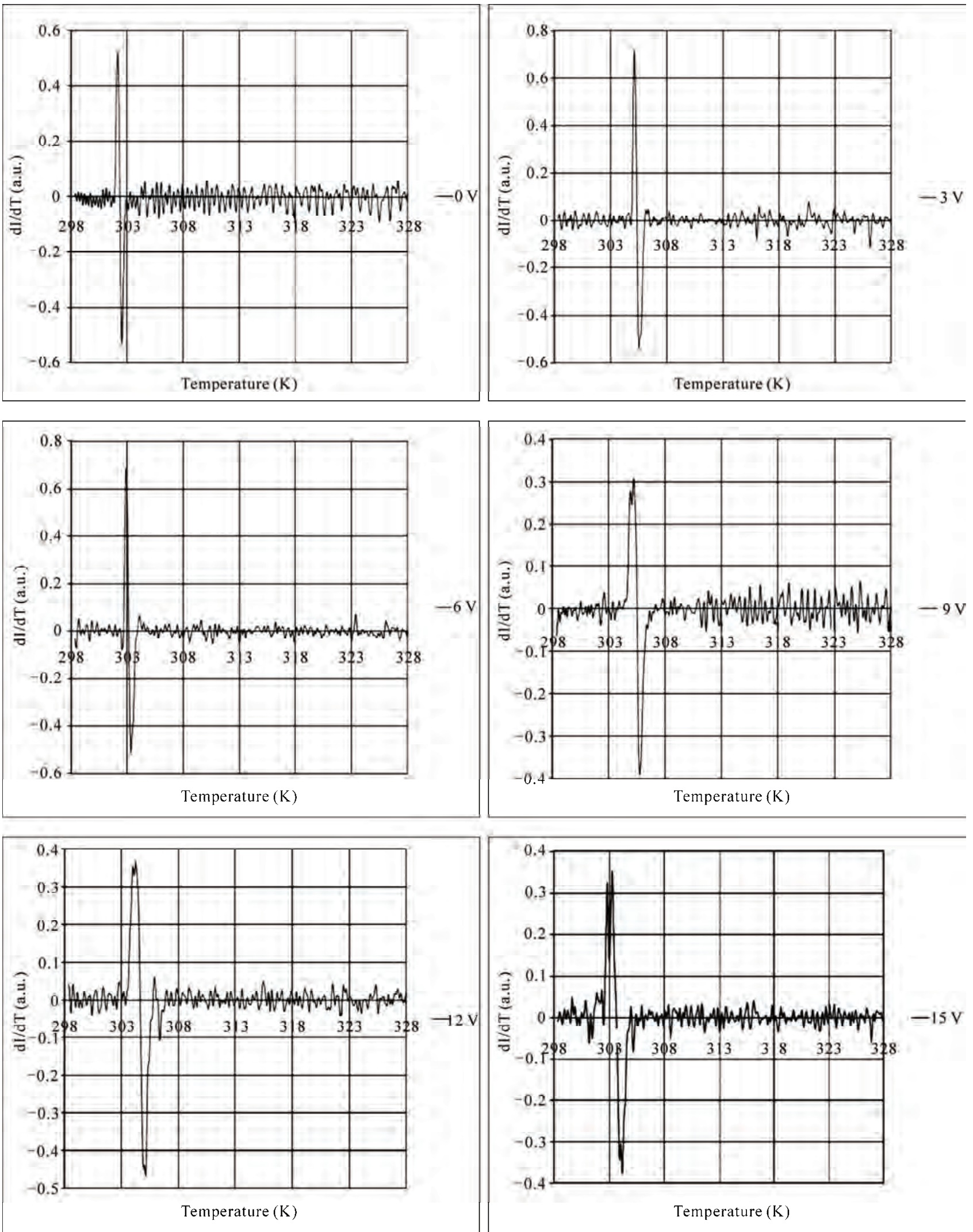

Figure 5. The modulation spectrum of the optical transmittance versus the temperature variations for the planar orientation by mechanical rubbing. 

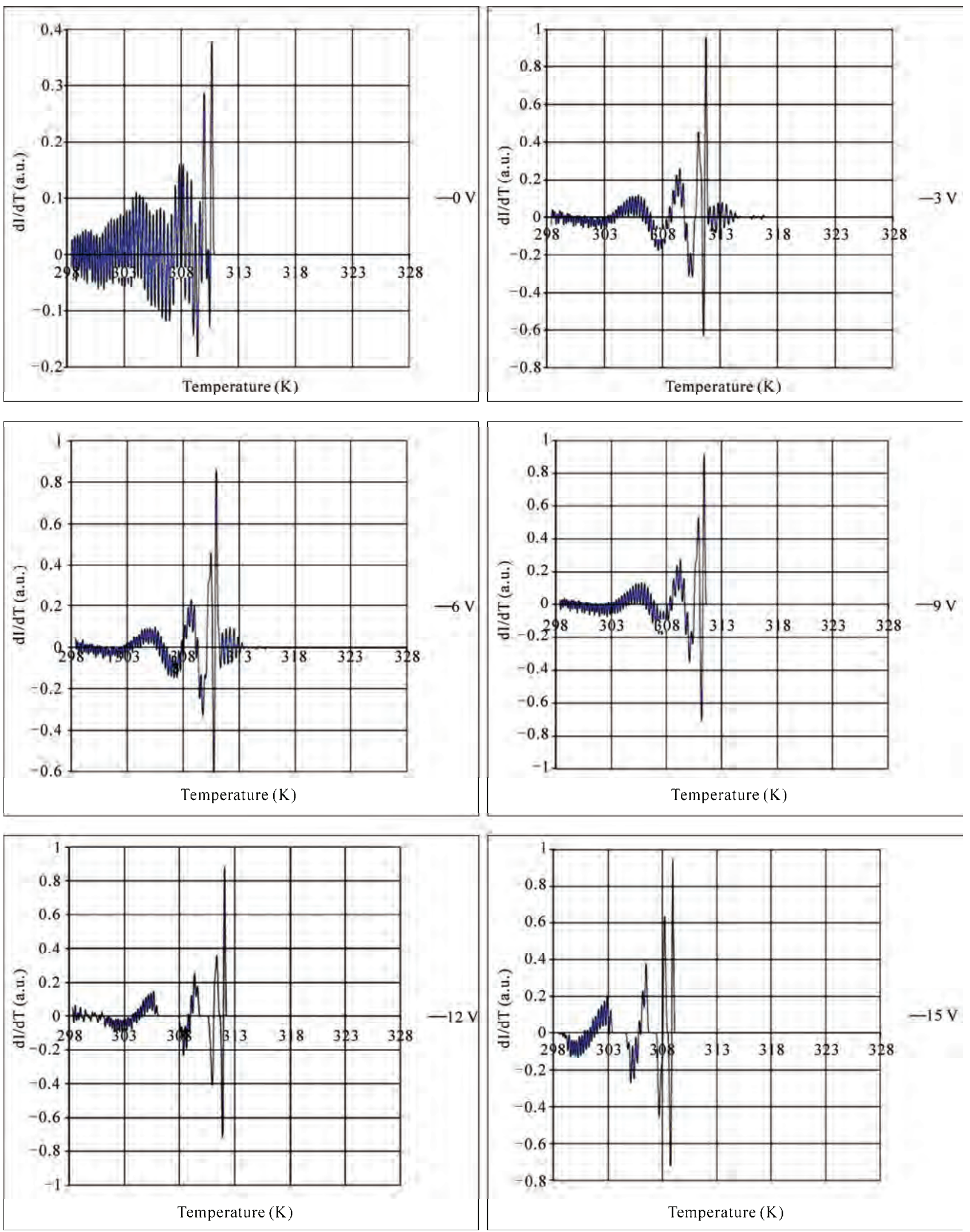

Figure 6. The modulation spectrum of the optical transmittance versus the temperature variations for the planar orientation by photolithography. 

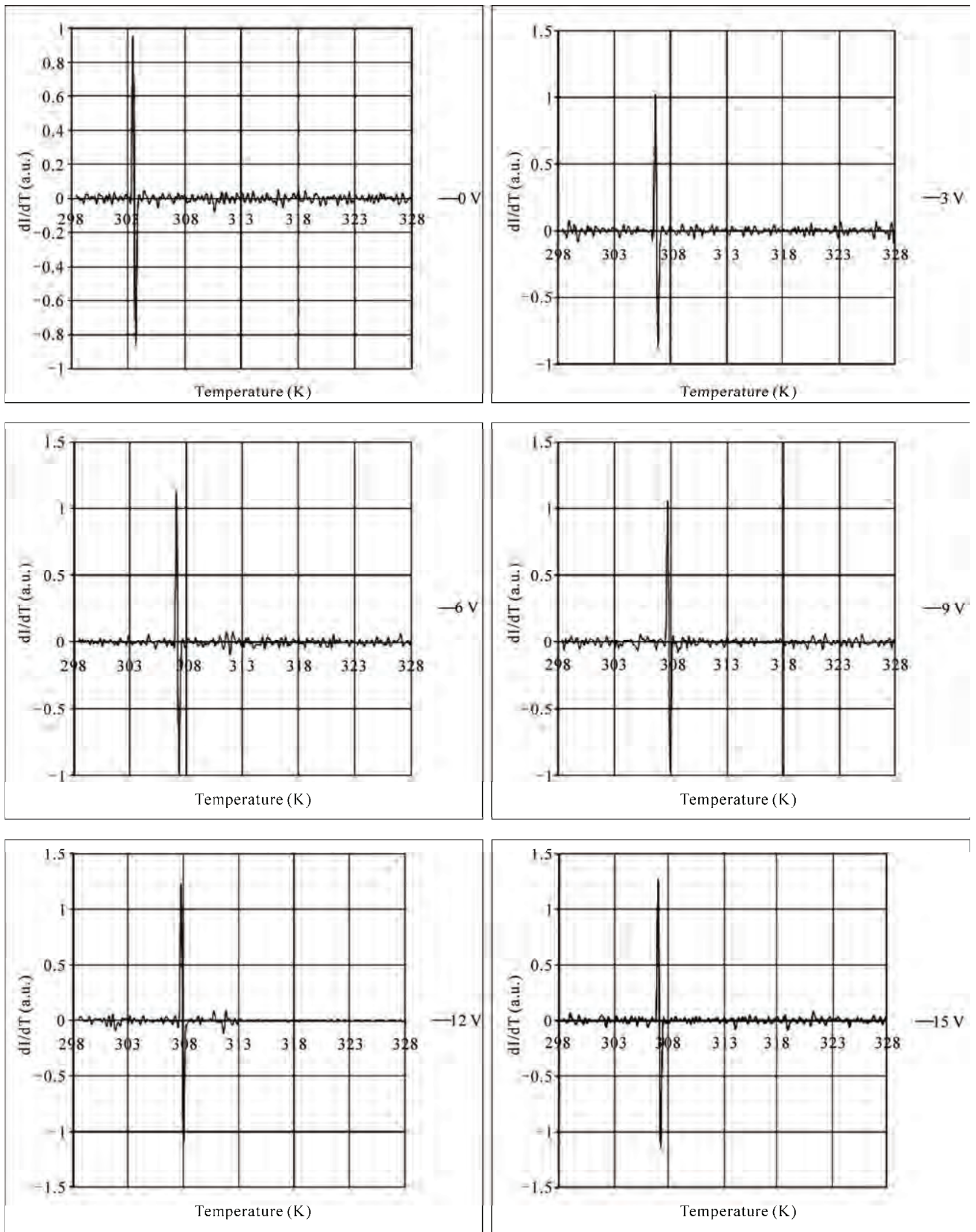

Figure 7. The modulation spectrum of the optical transmittance versus the temperature variations for the homeotropic orientation. 
The results of this study, which is accomplished on randomly orientated molecules in previous study [16], show resemblance with the results of other works by developed in the past studies [17-24].

\section{Acknowledgements}

The authors thank to Harran University for financial support. This study is supported by Harran University, the Fund of Scientific Research Projects (SRP) with grant no: 919 .

\section{References}

[1] S. Varghese, S. Narayanankutty, C. W. M. Bastiaansen, G. P. Crawford and D. J. Broer, "Patterned Alignment of Liquid Crystals by m-Rubbing," Advanced Materials, Vol. 16, 2004, pp. 1600-1605. doi:10.1002/adma.200306536

[2] V. A. Konovalov, V. G. Chigrinov, H. S. Kwok, H. Takada and H. Takadsu, "Photoaligned Vertical Aligned Nematic Mode in Liquid Crystals," Japanise Journal of Applied Physics, Vol. 43, No. 1, 2004, pp. 261-266. doi:10.1143/JJAP.43.261

[3] P. G. de Gennes and J. Prost, "The Physics of Liquid Crystals,” Oxford University Press, UK, 1993.

[4] H. Gleeson, "Introduction to Liquid crystals," Manchester University Press, UK, 1998.

[5] I. C. Khoo, "Liquid Crystals: Physical Properties and Nonlinear Optical Phenomena,” John Wiley \& Sons, Inc., New York, 1995.

[6] P. Klysubun, "Nonlinear Optical Studies of Dye-Doped Nematic Liquid Crystals,” PhD Dissertation, Virginia Polytechnic Institute and State University, Virginia, 2002.

[7] S. Yilmaz, and A. Bozkurt, "Spectroscopic Measurement of Liquid Crystal Anisotropy in the Ultraviolet/Visible Region,” Materials Chemistry and Physics, Vol. 107, No. 2-3, pp. 410-412.

[8] V. G. Chigrinov, "Liquid Crystal Devices: Physics and Applications,” Artech House, Boston, 1999.

[9] M. Emek, N. Besli, A. Yildirim and S. Yilmaz, "Optical Properties of Nematic Liquid Crystal $\left(\mathrm{C}_{21} \mathrm{H}_{27} \mathrm{NO}_{2} \mathrm{~S}\right)$ Under AC/DC Electric Fields" Canadian Journal of Physics, Vol. 87, No. 4, 2009, pp. 293-298. doi:10.1139/P09-030

[10] P. Yeh, and C. Gu, "Optics of Liquid Crystal Display," John Wiley \& Sons, New York, 1999.

[11] S. Kumar, "Liquid Crystals: Experimental Study of Physical Properties and Phase Transitions,” Cambridge University Press, Cambridge, 2001.

[12] J. Osterman, L. Madsen, J. Birgerson and K. Skarp, “Optical Properties of Single-Polarizer Reflective Bistable Nematic Displays," SID Symposium Digest of Technical Papers, Vol. 34, 2003, pp. 950-953. doi:10.1889/1.1832441
[13] D. Kang, J. E. Maclennan, N. A. Clark, A. Zakhidov and R. H. Baughman, "Electro-Optic Behavior of LiquidCrystal-Filled Silica Opal Photonic Crystals: Effect of Liquid-Crystal Alignment," Physical Review Letters, Vol. 86, No. 18, 2001, pp. 4052-4055.

doi:10.1103/PhysRevLett.86.4052

[14] S. Yilmaz, "Light Scattering By Some Nematic Liquid Crystals Due to Phase Transitions," Journal of Quantative Spectroscopy and Radiative Transfer, Vol. 104, No. 3, 2007, pp. 305-309. doi:10.1016/j.jqsrt.2006.02.002

[15] J. Osterman, "Investigations of Optical Properties and Photo-Alignment in Bistable Nematic Liquid Crystal Displays,” PhD Dissertation, Uppsala University, Sweden, 2005.

[16] S. Yilmaz, "Optical Properties of Some Mixed Nematic Liquid Crystals in Electric Field,” Materials Chemistry and Physics, Vol. 110, No. 1, 2008, pp. 140-144. doi:10.1016/j.matchemphys.2008.01.027

[17] Y. Suzuki, N. Mizoshita, K. Hanabusa and T. Kato, "Homeotropically Oriented Nematic Physical Gels for Electrooptical Materials,” Journal of Materials Chemistry, Vol. 13, No. 12, 2003, pp. 2870-2874.

doi:10.1039/b308098f

[18] R. Bartolino, N. Scaramuzza, D. E. Lucchetta, E. S. Barna, A.Th. Ionescu and L.M. Blinov, "Polarity Sensitive Electrooptical Response in a Nematic Liquid CrystalPolymer Mixture,” Journal of Applied Physics, Vol. 85, No. 5, 1999, pp. 2870-2874. doi:10.1039/b308098f

[19] H. Y. Chen and W. Lee, "Electro-Optical Characteristics of a Twisted Nematic Liquid-Crystal Cell Doped with Carbon Nanotubes in a DC Electric Field," Optical Reviews, Vol. 12, No. 3, 2005, pp. 223-225. doi:10.1007/s10043-005-0223-7

[20] J. G. Yoo, B. S. Song and J. Kim, "Surface Pretilt Effects of Bistable Twisted Nematic Liquid Crystal Display," Japanise Journal of Applied Physics, Vol. 38, No. 10, 1999, pp. 6005-6007. doi:10.1143/JJAP.38.6005

[21] H. L. Margaryan and V. M. Aroutiounian, "Influence of Longitudinal Electric Field on Time Characteristics of Freedericksz Transition,” Moleculer Crystals and Liquid Crystals, Vol. 454, No. 1, 2006, pp. 513-523.

[22] J. W. Han, "Effect of the Nematic-Isotropic Phase Transition on the Electro-Optical Characteristics of PolimerDispersed Liquid Crystal Films,” Journal of the Korean Physical Society, Vol. 40, No. 5, pp. 849-855.

[23] T. Takematsu, H. Okada and H. Onnagawa, "Characteristics of Modulated Microdomain Switching Using Nematic Liquid Crystals,” Japanese Journal of Applied Physics, Vol. 41, No. 2A, 2002, pp. 733-736. doi:10.1143/JJAP.41.733

[24] J. Merlin, E. Chao, M. Winkler and K. D. Singer, P. Korneychuk and Y. Reznikov, "All-Optical Switching in a Nematic Liquid Crystal Twist Cell,” Optics Express, Vol. 13, No. 13, 2005, pp. 5024-5029.

doi:10.1364/OPEX.13.005024 